http://jmscr.igmpublication.org/home/

ISSN (e)-2347-176x ISSN (p) 2455-0450

crossref DOI: https://dx.doi.org/10.18535/jmscr/v8i2.34

\title{
The Clinical and Biochemical Profile of Patients with Urolithiasis coming to the Nephrology Department of this Tertiary Care Centre in South India
}

\author{
Authors \\ Akshaj Manilal ${ }^{1}$, Dr Kishore S Dharan², Dr Anna Mathew ${ }^{3^{*}}$, Mr. Prakash Ramasami \\ ${ }^{1}$ Interne MOSC Medical College, Kolenchery, Kerala 682311 \\ ${ }^{2}$ Associate Professor, Department of Nephrology, MOSC Medical College, Kolenchery, Kerala 682311 \\ ${ }^{3}$ Professor and HOD, Department of Pharmacology, MOSC Medical College, Kolenchery, Kerala 682311 \\ ${ }^{4}$ Consultant Biostatistician \\ *Corresponding Author \\ Dr Anna Mathew
}

Professor and HOD, Department of Pharmacology, MOSC Medical College, Kolenchery, Kerala 682311

\begin{abstract}
Background: Urolithiasis is one of the most important causes of acute and chronic urinary failure.presenting with acute renal colic, severe loin pain, frequent urination, dysuria, oliguria and haematuria. It can be precipitated by dehydration and reduced urine output, increased protein intake, heavy physical exercise, and various medicines. The treatment of urolithiasis involves emergency management of renal (ureteral) colic, including surgical interventions where indicated, and medical therapy for stone disease. This cross-sectional study was undertaken to find the prevalence of urolithiasis in patients presenting with acute kidney injury and to study the biochemical parameters associated with urolithiasis.
\end{abstract}

Objectives: To find the prevalence of renal urolithiasis presenting with acute kidney injury and to document the clinical findings, risk factors and outcomes of urolithiasis in patients coming to the nephrology department of this tertiary care hospital.

Methodology: After approval for study was obtained from the Institutional Review Board and Ethics Committee data for this cross-sectional study was collected from the medical records of patients admitted for urolithiasis in this institution from April 2016. The sample size was calculated to be 246, for single proportion, for a confidence interval of $95 \%$ and precision of $5 \%$ using the estimated prevalence of urinary stones of $20 \%$. The data was analysed using SPSS software to find the prevalence and risk factors of Urolithiasis in acute kidney injury.

Results and Conclusions: In our study we found urolithiasis was commonly seen in male patients between the ages of 50 and 60 years, who presented commonly with flank pain and vomiting. Serum creatinine was elevated in around two thirds of the patients and the commonest feature on urine analysis was haematuria. Most of the patients (70\%) were surgically managed. The prevalence of acute kidney injury due to urolithiasis was $23 \%$ and chronic kidney disease was $3 \%$.

Keywords: Urolithiasis, kidney stones, calculi, lithotripsy, acute kidney injury. 


\section{Introduction}

Urolithiasis is a common problem with a worldwide estimated prevalence of $20 \%$ and a 5year recurrence rate of $50 \%$ and the incidence and prevalence of kidney stones is increasing globally. ${ }^{[1]}$ It is one of the most important cause of acute and chronic urinary failure and thus results in high morbidity and enoromous socio-economic burden. ${ }^{[2]}$

The epidemiology of nephrolithiasis varies according to the geographical area \& socio economic conditions. A study from Coimbatore reported that there was a considerable increase in incidence of kidney stones in Coimbatore from 1969 to 1984 and that $5-12 \%$ of the population will develop kidney stones during their life time. ${ }^{[3]}$

The mechanism of stone formation begins with supersaturation of the urine with salts, nucleation (forming nuclei), crystal growth and agglomeration. Risk factors for formation of stones include urinary promoters (calcium, urate, cystine, and sodium) and urinary inhibitors (magnesium, citrate, and nephrocalcin). Daily dietary oxalate was found to be much higher in people who developed stones and the main sources of oxalate in diets were regular tea and coffee. ${ }^{[4]}$ Others have reported higher intake of sodium chloride among stone formers ${ }^{[3]}$ and higher intake of animal proteins, oxalate, sodium, a low intake of fluids and potassium containing citrus products. ${ }^{[5]}$

Symptoms include acute renal colic, manifested by severe loin pain, frequent urination, dysuria, oliguria and haematuria, which may be precipitated by dehydration and reduced urine output, increased protein intake, heavy physical exercise, and various medications. ${ }^{[6]}$

Nutrition is a key determinant in calculi formation. The increase in frequency of calculi is closely related to the genetic and environmental factors such as fluid intake, low urinary volume and high urine concentration due to warm climate, immobilization, occupation and certain medications. Metabolic disorders, heredity, water supply, alcohol consumption and smoking often have been blamed for calculi formation. ${ }^{[7]} \mathrm{A}$ systematic review revealed that individuals who

had normal calcium intakes, low intake of oxalates, dietary proteins and salt had significantly reduced rate of calcium oxalate stone recurrence. ${ }^{[8]}$ A study from Tamilnadu (1997) studied 100 patients with kidney stones and found 96 patients used tobacco, betel leaves with churum containing calcium carbonate, tea or coffee, smoked and consumed alcohol. They reported that $84 \%$ patients consumed less than one litre of water per day, which is another causative factor of urinary calculi. ${ }^{[9]}$

The treatment of urolithiasis involves emergency management of renal (ureteral) colic, including surgical interventions where indicated, and medical therapy for stone disease. This cross-sectional study on patients with urolithiasis was planned to answer the research question, "What is the prevalence of acute kidney injury and the clinical profile and outcome of urolithiasis in patients presenting to the nephrology department of this institution?

Kidney stones are of two types, primary stones of calcium, oxalate, uric acid, cystine and xanthine and secondary stones that are formed by urea splitting organisms such as Proteus, Pseudomonas and Klebsiella species and are known as struvite stones. They are composed of magnesium, ammonium and phosphates. ${ }^{[10]}$ Most stones contain calcium combined with oxalate, phosphate or occasionally uric acid in the form of calcium oxalate, calcium phosphate, calcium carbonate, brushite, gypsum and/or dolomite. All calcium stones are radiopaque. Uric acid stones are radiographically transparent unless mixed with calcium crystals or struvite and, in contrast to the radiopaque calcium stones, they are radiolucent. Triple phosphate stones are crystalline struvite stone composed of magnesium ammonium phosphate and are also known as infection stones. ${ }^{[1]}$ Uric acid crystals can induce the development of calcium oxalate crystals on them through a heterogeneous nucleation process and some crystallization inhibitors like phytate and pyrophosphate can delay this process. ${ }^{[10]}$ 
Some medicines may enhance stone formation, and in the case of uric acid stones, such drugs include hyperuricosuric agents, such as low-dose salicylates, probenecid and thiazides. Indianavir sulphate, which is an HIV protease inhibitor, has been associated with urolithiasis insome patients. [12]

Diabetes mellitus is associated with an increased risk of kidney stone formation. The compensatory hyperinsulinemia of type 2 diabetes may increase the supersaturation of the urine with calcium salts. Studies have shown that increased glucose decreased the tubular reabsorption of filtered calcium and increased urinary calcium excretion. ${ }^{[13]}$ Insulin resistance might result in a deficit in ammonium production in the kidney, which lowers urinary $\mathrm{pH}$, thus generating a favorable milieu for uric acid stone formation. ${ }^{[14]}$ Studies have shown that insulin resistance is a predisposing factor leading to uric acid nephrolithiasis due to a low urinary ammonium and $\mathrm{pH}$. In addition to the reduced ammonium excretion associated with insulin resistance, there are additional mechanisms leading to the reduced ammonium excretion in uric acid stone formers. ${ }^{[15]}$ Because a growing percentage of our population has features of insulin resistance the prevalence of obesity, diabetes and the metabolic syndrome may be a reason for the surge in the population risk and incidence for kidney stones. ${ }^{[14,15,16]}$ Similar associations have been demonstrated with hypertension, dyslipidaemia,

cardiovascular diseases and metabolic syndrome ${ }^{[16,17,18]}$

Protocol based metabolic evaluation in high risk patients would reveal several metabolic abnormalities, including hypercalciuria, hyperoxaluria, hyperuricosuria, hypocitraturia, and hypomagnesuria, though stone formation may occur in the absence of any of these risk factors

Kidney damage is defined as pathologic abnormalities or markers of damage, including abnormalities in blood or urine tests or imaging studies. ${ }^{[11]}$ Renal function is assessed using the serum creatinine levels and urinary output to determine if patients have normal renal function, acute kidney injury or chronic renal failure. The classification of Acute Kidney Injury and Chronic Kidney Disease is done according to the National Kidney Foundation Kidney Disease Outcomes Quality Initiative classification. ${ }^{[19]}$

Chronic Kidney disease (CKD) is defined as either kidney damage or glomerular filtration rate (GFR) less than $60 \mathrm{~mL} /$ minute per $1.73 \mathrm{~m}^{2}$ for three or more months.

\section{Acute Kidney Injury Network (AKIN)} classification 2005 for staging acute kidney injury, This is a highly sensitive staging system and is based on recent data indicating that a small change in serum creatinine influences outcome. Only one criterion (creatinine or urine output) must be fulfilled to qualify for a stage on the AKIN staging system. ${ }^{[20]}$

Table 1 The AKIN Classification/staging system of Acute Kidney Injury

\begin{tabular}{|l|l|l|}
\hline Stage & Serum Creatinine Critria & Urine Output criteria \\
\hline 1. & $\begin{array}{l}\text { Increase of serum creatinine to } \geq 0.3 \mathrm{mg} / \mathrm{dL}(\geq 26.5 \\
\mu \mathrm{mol} / \mathrm{L}) \text { or } \geq 150 \text { to } 300 \%(1.5 \text { to } 2 \text {-fold })\end{array}$ & Less than $0.5 \mathrm{ml} / \mathrm{kg}$ per hour for more than 6 hours \\
\hline 2. & $\begin{array}{l}\text { increase of serum creatinine from baseline Increase } \\
\text { of serum creatinine }>200 \text { to } 300 \%(2-3 \text {-fold }) \text { from } \\
\text { baseline }\end{array}$ & Less than $0.5 \mathrm{ml} / \mathrm{kg}$ per hour for more than 12 hours \\
\hline 3. & $\begin{array}{l}\text { Increase of serum creatinine to more than } 3 \text {-fold } \\
\text { from baseline (serum creatinine of } \geq 4.0 \mathrm{mg} / \mathrm{dL}(\geq 354 \\
\mu \mathrm{mol} / \mathrm{L}) \text { with an acute increase of at least } 0.5 \mathrm{mg} / \mathrm{dL} \\
(44 \mu \mathrm{mol} / \mathrm{L})\end{array}$ & $\begin{array}{l}\text { Less than } 0.3 \mathrm{ml} / \mathrm{kg} \text { per hour for } 24 \text { hours or anuria } \\
\text { for } 12 \text { hours }\end{array}$ \\
\hline
\end{tabular}

Mehta RL, Kellum JA, Shah SV et al. Acute Kidney Injury Network: report of an initiative to improve outcomes in acute kidney injury. Crit Care 2007; 11: R31.17 ${ }^{[17]}$

This study was planned to document the historical, with urolithiasis to this tertiary care hospital to clinical and biochemical profile of patients coming assess the outcomes in these patients. We also 
wanted to find the prevalence of acute kidney injury and chronic renal failure in patients presenting with urolithiasis.

\section{Materials and Methods}

After ethical committee approval was obtained, patients over 18 years of age, with a diagnosis of urolithiasis were enrolled into this cross-sectional study from the medical records of the urology department of this tertiary care centre. The details of standard clinical examinations, routine biochemical and haematological investigations and treatment were collected.

The sample size was found to be 246 , for a confidence interval of $95 \%$ and precision of $5 \%$. The Sample size for single proportion of 0.2 was calculated using nMaster computer software, using the prevalence of urinary stones of $20 \%$ reported by Sharma et al. ${ }^{[2]}$

Assessment of renal function was done using the guidelines of the National Kidney Foundation for chronic renal failure and acute kidney injury by the AKIN classification. The risk factors assessed included were diet, drug use, alcohol and smoking. The management was either conservative or surgical and we have documented the various interventions undertaken in these patients. The prevalence of urolithiasis presenting as acute or renal failure was obtained. The study flowchart following Strobe guidelines for observational studies is given in Figure 1.

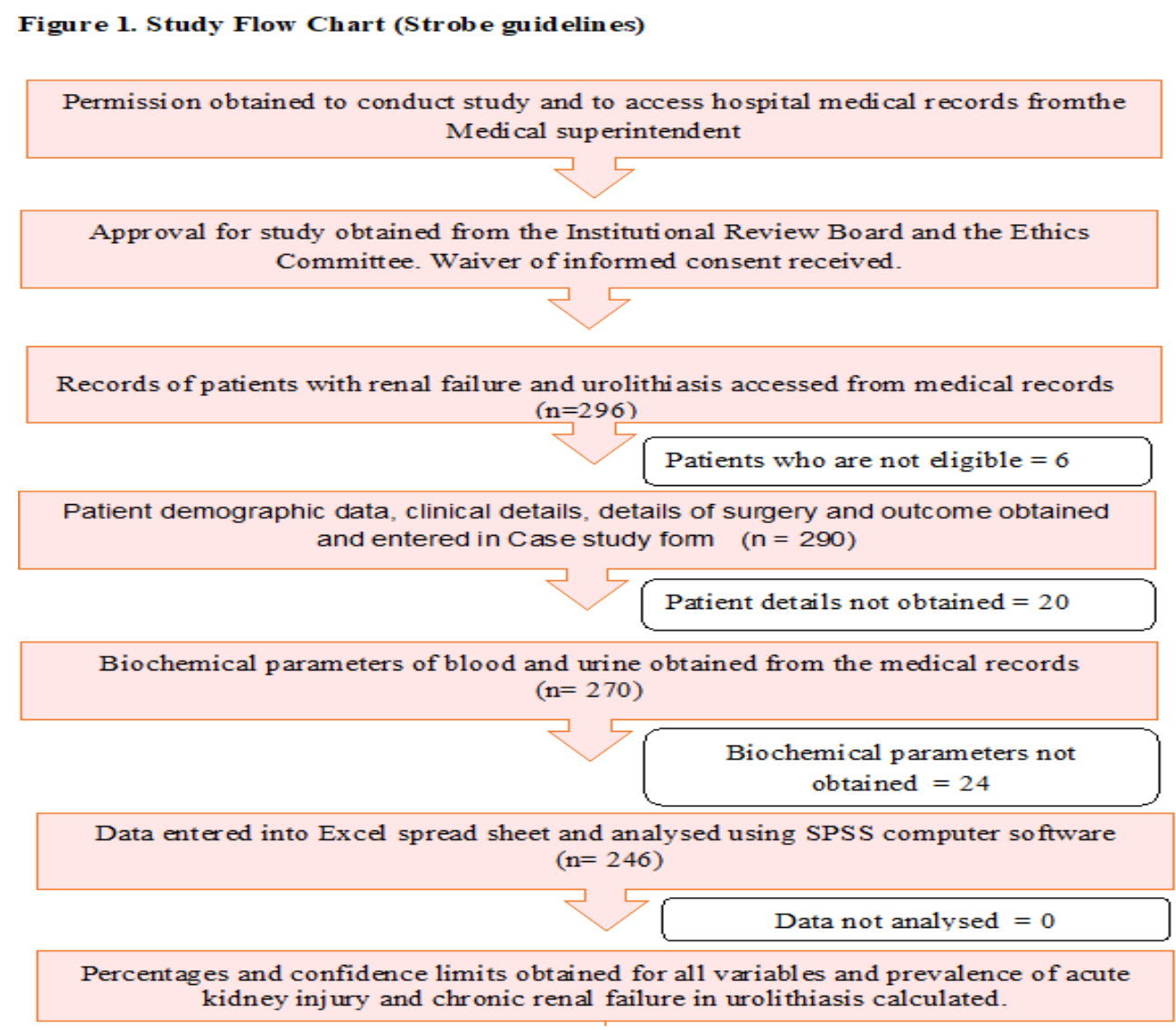

\section{Results}

The mean age of the 246 participants included in this study was 44.1 (SD 14.5) years of whom nearly $70 \%$ were in the age group 30 to 59 years, Around $80 \%$ were males and two thirds of the patients came from a rural setting. The occurrence of urolithiasis increases steadily till the fourth and fifth decade and then declines. The maximum number of people (24\%) were affected in the fourth and fifth decade of life. Recurrent disease was found in $89(36 \%)$ of the 246 participants. 
Table 1: Baseline Characteristics of the Study Sample ( $n=246)$

\begin{tabular}{|lc|c|}
\hline Baseline Characteristics of the participants & Number (\%) \\
\hline Age & $<29$ years & $36(14.6 \%)$ \\
& $30-59$ years & $169(68.7 \%)$ \\
\hline Gender (n=246) & $>60$ years & $41(16.7 \%)$ \\
\hline Hometown (n=246) & Male & $195(79.3 \%)$ \\
& Female & $51(20.7 \%)$ \\
\hline Recurrent stone disease & Rural & $164(66.7 \%)$ \\
& Urban & $82(33.3 \%)$ \\
\hline Clinical findings present & Yes & $89(36.2 \%)$ \\
& No & $157(83.8 \%)$ \\
\hline Symptoms & Yes & $111(45.1 \%)$ \\
& No & $135(54.9 \%)$ \\
& Flank Pain & $189(76.8 \%)$ \\
& Dysuria & $45(18.3 \%)$ \\
& Hematuria & $29(11.8 \%)$ \\
& Fever & $29(11.8 \%)$ \\
& Acute Urinary Retention & $10(4.1 \%)$ \\
\hline Co-Morbidities & Nausea and Vomiting & $94(38.2 \%)$ \\
\hline Urine analysis & Diabetes Mellitus & $63(25.6 \%)$ \\
& Cardiovascular diseases & $59(24 \%)$ \\
& Renal Diseases & $7(2.8 \%)$ \\
\hline & Albuminuria & $21(8.1 \%)$ \\
& Calcium Oxalate Crystals & $16(6.5 \%)$ \\
& Pyuria & $77(31.3 \%)$ \\
& Hematuria & $115(45.7 \%)$ \\
\hline
\end{tabular}

\section{Clinical Features}

The most characteristic symptom was flank pain $189(76.8 \%)$ followed by nausea and vomiting (38\%). Clinical findings were elicited in $45 \%$ of the participants. Diabetes mellitus and cardiovascular disease were the commonest comorbidities.

\section{Biochemical Parameters}

The serum creatinine was elevated in $64(26 \%)$ of the participants in this study. Elevated serum uric acid was found in seven of the 22 patients for whom the test was done. Of the 246 participants, the urine showed microscopy showed pus cells in $77(31.3 \%)$ subjects, significant albuminuria was present in $21(8.1 \%)$ participants and calcium oxalate crystals were identified in $16(6.5 \%)$ participants. The commonest urine analysis finding was haematuriain $115(45.7 \%)$ participant of whom $86(74.8 \%)$ had microscopic haematuria and $29(26.2 \%)$ had macroscopic haematuria.

\section{Location of Calculi}

Of the 246 participants, $198(80.5 \%)$ had bilateral calculi, while $48(19.5 \%)$ had unilateral calculi. A total of 300 calculi were recorded in the 246 participants. The most common type of urolithiasis was ureteric calculi, accounting for about $45 \%$ of the calculi while the least common was urethral calculi which was only $2 \%$, while renal calculi accounted for $35 \%$ and vesical and vesico-urethral junction calculi $15 \%$. Renal calculi accounted for about $43 \%$ and 8 subjects had staghorn calculi. The sites of location of the calculi are shown in figure 1. 


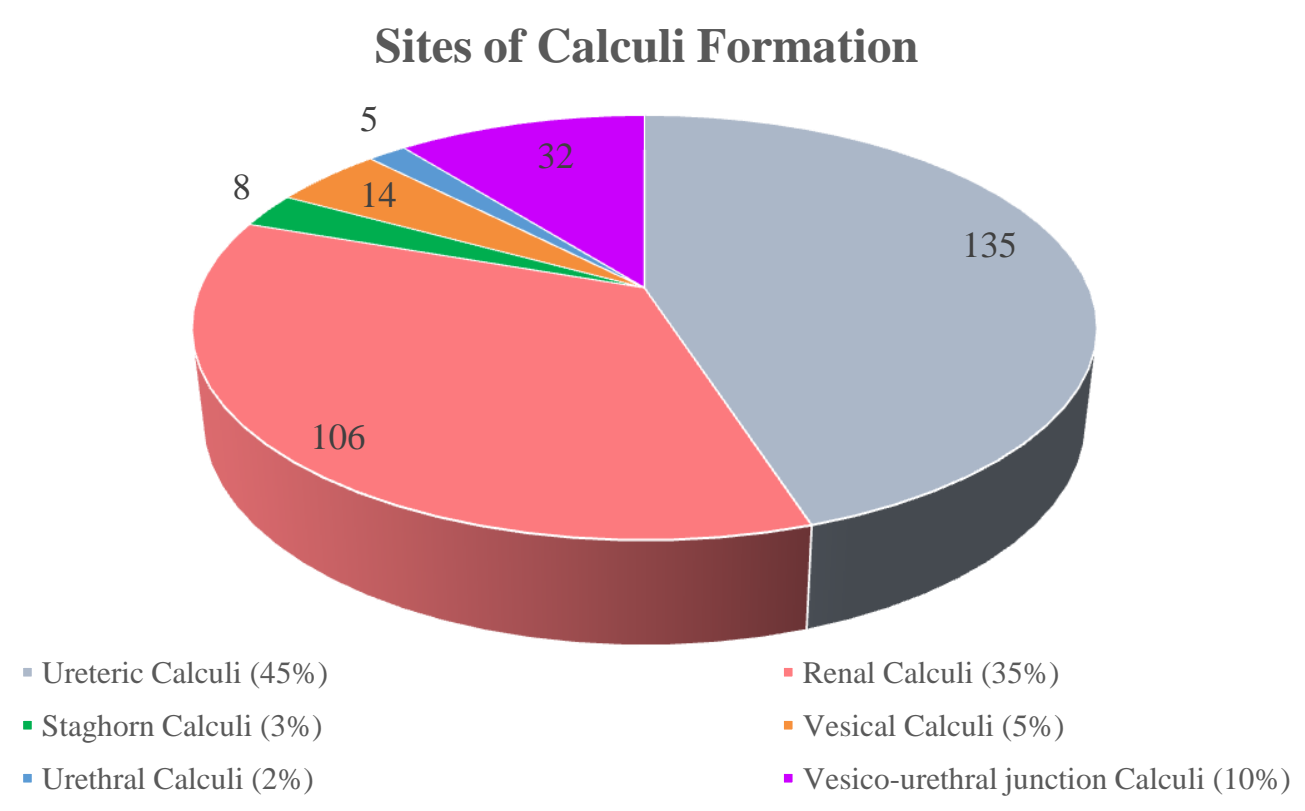

Figure 2. Frequency Distribution of Sites of Calculi Formation in Participants

Legend: Of the 300 calculi diagnosed in the 246 patients, 135 (45\%)were located in the ureters; 106 (35\%) were renal, of which $8(3 \%)$ were staghorn; $32(10 \%)$ were located in the vesico-ureteric junction; $14(5 \%)$ were in the bladder and $5(2 \%)$ werein the urethra

\section{Sequelae of Urolithiasis}

Of the 246 patientswith urolithiasis, only 17 $(6.9 \%)$ did not develop any sequelae in the kidney.
Of the remaining 229 (93.1\%) who had some form of kidney damage, 57 (23.2\%) had acute kidney injury and $8(3.3 \%)$ chronic kidney disease, 114 (46.3\%) had hydroureteronephrosis and 50 $(20.3 \%)$ had hydronephrosis. as illustrated in figure 3 . Of the 57 patients who developed acute kidney injury, 49 (86\%) were in stage 1 AKI as per AKIN classification. The sequelae of urolithiasis on the kidneyas illustrated in figure 3.

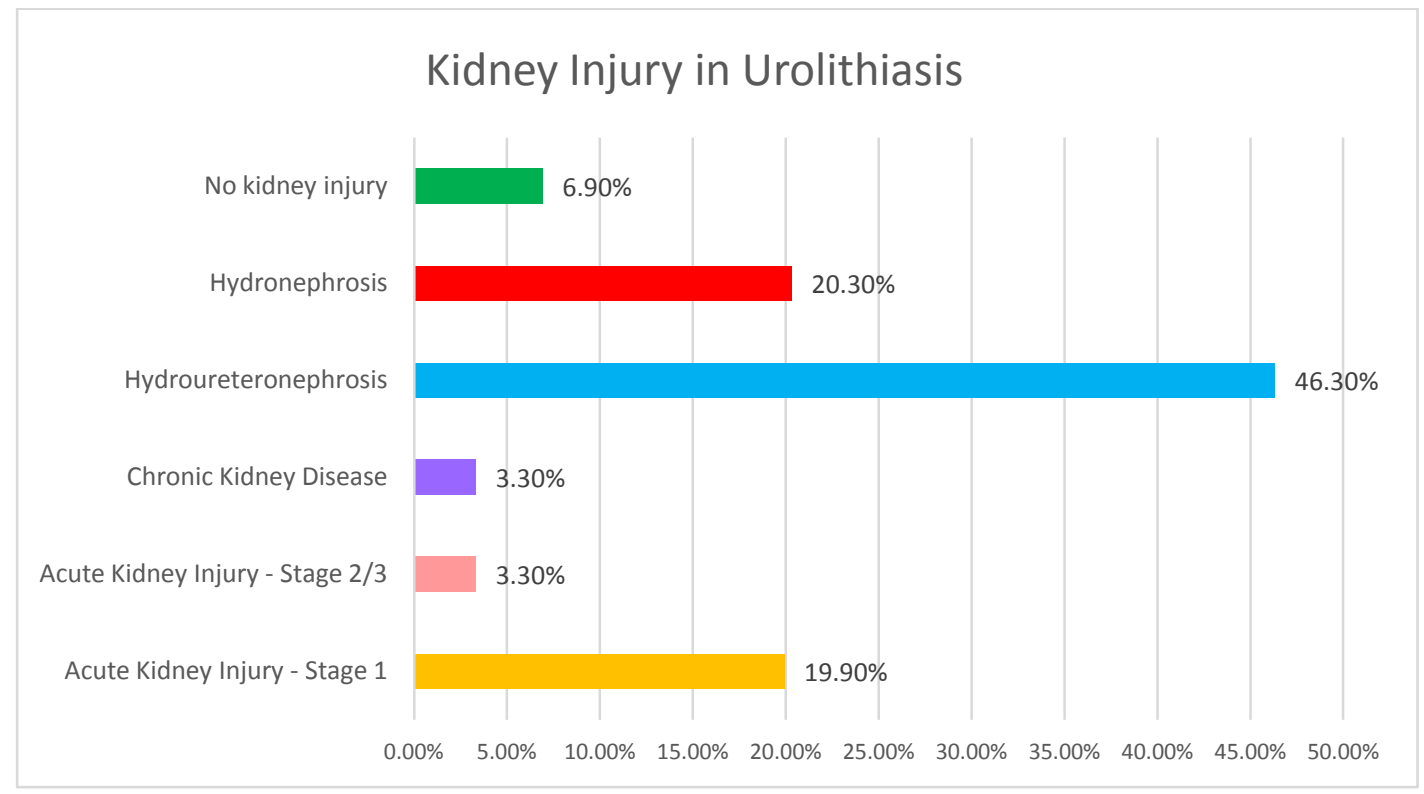

Figure 3. Frequency of Sequalae of Urolithiasis

Legend: Most of the patients who had urolithiasis $(93.1 \%)$ had developed some form of sequalae and only $6.9 \%$ had no kidney injury. The prevalence of acute kidney injury in patients with urolithiasis was $23.2 \%$ and of these nearly $20 \%$ were in Stage 1 according to the AKIN classification. 


\section{Management Outcomes}

Surgical management was undertaken in 173 $(70.3 \%)$ of the patients, while $72(29.3 \%)$ were managed conservatively and one patient was discharged against medical advice. Double J stenting (DJS) together with Ureteroscopic Lithotripsy (URSL) was done in 91(52.6\%). DJS and URSL and together with Extracorporeal Shockwave Lithotripsy (ESWL) of patients in 25 (14.5\%). The other interventions were and Per Cutaneous Lithotripsy (PCNL) and cystolithotripsy and one patient had a nephrectomy,

The various interventions performed is shown in figure 4.

\section{Surgical Interventions Used in Patients with Urolithiasis}

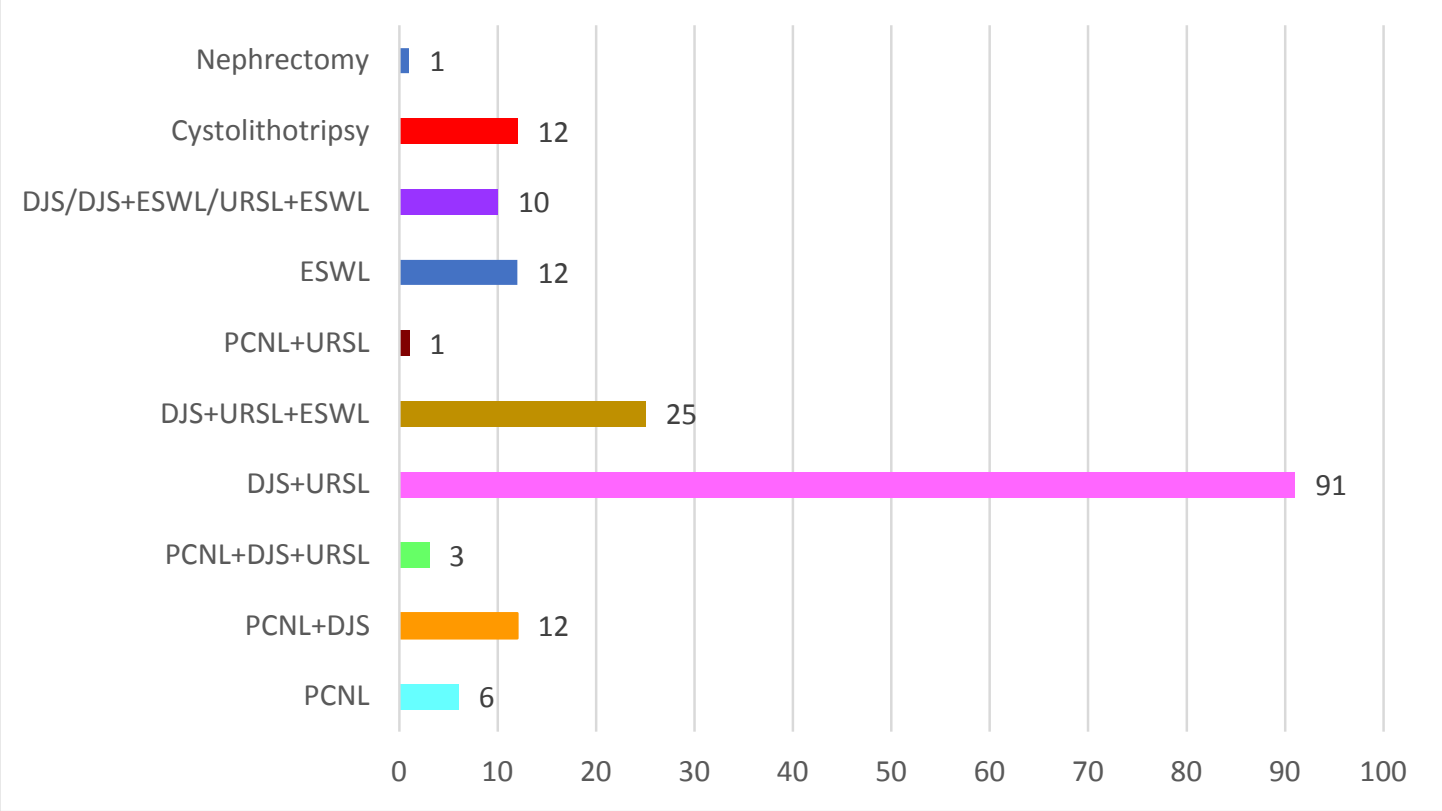

Figure 4. Management of Patients with Urolithiasis

PCNL $\rightarrow$ Percutaneous Nephrolithotomy DJS $\rightarrow$ Double J stenting lithotripsy

ESWL $\rightarrow$ Extra corporeal shock wave URSL $\rightarrow$ Ureteroscopic lithotripsy

Legend: Majority of the patients were surgically managed (70\%) while the remaining $30 \%$ were medically managed

\section{Discussion}

We studied the profile of 246 adults who were diagnosed to have urolithiasis. Most of the patients were male and came from a rural setting. In our study the majority $(81.7 \%)$ of patients were The mean age of the sample in our study was 44.1 ( \pm $14.5)$ years. The youngest patient selected was of the age 18 years and oldest was of the age 78 years old. The occurrence was only $37 \%$ among the age group 20-40 while it was $68.7 \%$ in age group 3060 years of age. Bharathi and Amirthaveni (2007) found the average age at first stone formation was $37.7+12.5$ years. The occurrence was $59.9 \%$ among 20-40 years of age. The youngest stone patient selected was of 5 years and oldest was 82 years old. ${ }^{[3]}$ Our study showed the incidence of urolithiasis peaked during the fourth and fifth decade of life with about 119 subjects $(48.4 \%)$ of total study population followed by a decline in incidence.

The study from Coimbatore also reported that most patients were in the same age group. ${ }^{[3]}$ The peak age for onset of stone formation is in the third decade, and prevalence increases with age until 70 . During the past few decades, the prevalence of kidney stones in both males and females has markedly increased in industrialized countries. This is presumably due to changes in lifestyle and dietary habit of the people in these regions. ${ }^{[18]}$ 
Most of the patients presented with flank pain(76.8\%), vomiting(38.2\%), dysuria, haematuria, fever $(11.8 \%)$ or acute urinary retention, though clinical findings were manifest in only $45 \%$ of the patients. Bharathi et al foundhaematuria was a common symptom while in our study only around $12 \%$ of patients presented with haematuria. The study from Tamil Nadu reported other urinary symptoms including incomplete voiding (72\%),haematuria (43\%), burning micturition $(6 \%)$ and oliguria $(16 \%) .{ }^{[9]}$

Bharathi and Amirthaveni (2007) reported that among 700 stone formers of Tamil Nadu 89.60 per cent were of low and middle income groups. Nearly 23.70 per cent of female stone formers and 28.90 per cent of male stone formers were illiterate. $54 \%$ of them were educated up. Only $18.7 \%$ had higher education and $16.4 \%$ had the family history of kidney stones. ${ }^{[3]}$ Out of these 321 were males and 28 were females.

Diabetes mellitus was also present in around 25\% of the participants of the study and cardiovascular disease in $25 \%$. In our study of the 63 diabetic patients with urolithiasis, significantly more developed renal failure 38 (60.3\%) developed renal failure $(\mathrm{p}=0.006)$.

Regarding biochemical parameters, we found in our study that serum creatinine was elevated in 64 (26\%) of the subjects in this study. The commonest urine analysis findings was haematuria, seen in over $45 \%$ of subjects and among these $75 \%$ had microscopic haematuria and the remaining were macroscopic haematuria. Abate et al found that a renal manifestation of insulin resistance may be low urinary ammonium and $\mathrm{pH}$. This defect can result in increased risk of uric acid precipitation despite normouricosuria. In our study we were not able to obtain urinary ammonium values for diabetic patients. This will be done in future studies in diabetic patients. ${ }^{[16]}$ The most common type of urolithiasis was ureteric calculi which accounted for about $68 \%$ of the stones in our study population. Staghorn calculus was seen in 8 Subjects. Stones were found unilaterally in about $79 \%$ of the subjects while in $21 \%$ of subjects it was bilateral. Sharma et al in a South Indian study found renal stones were most common, followed by ureteric and bladder stones and that calcium and oxalate were the most common constituents of urinary stones. ${ }^{[2]}$

Regarding outcomes, most of the patients in our study were surgically managed $(70 \%)$ while only the remaining $30 \%$ were managed conservatively. The commonest surgical procedure performed was double J stenting, which was done in almost $80 \%$ of patients. Ureteroscopic lithotripsy was done in more than $70 \%$ of patients. Extracorporeal shockwave lithotripsy was done in $26 \%$ of patients and $12 \%$ underwent percutaneous nephrolithotomy and around 75 underwent cystolithotripsy.

We observed that the prevalence of acute kidney injury in patients with urolithiasis was $23 \%$ and chronic kidney disease was 3\%. Of the patients who presented with acute kidney injury $86 \%$ were in stage $1 \mathrm{AKI}$ as per AKIN classification. More than $45 \%$ of subjects had hydroureteronephrosis and 20\% had hydronephrosis. Of the 137 patients with hydroureteronephrosis (HUN)/hydronephrosis (HN), $103(41.2 \%)$ were surgically treated with Double J stenting while only 35 had other procedures. $(\mathrm{p}=0.006)$.

\section{Conclusion}

In this study we found urolithiasis was commonly seen in male patients between the ages of fifty and sixty who presented commonly with flank pain and vomiting. Serum creatinine was elevated in around two thirds of the patients and the commonest feature on urine analysis was haematuria. Most of the patients (70\%) were surgically managed and Double J stenting (DJS) together with ureteroscopic lithotripsy (URSL) was done in over $50 \%$ of the patients. The prevalence of acute kidney injury due to urolithiasis was $23 \%$ and chronic kidney disease was $3 \%$.

Renal calculi is a common problem in this area with the prevalence of renal calculi being $20 \%$ in this part of the country. The prevalence of acute kidney injury in patients with urolithiasis was $23 \%$ and chronic kidney disease was $3 \%$. This study has 
detailed the clinical and biochemical profile of patients with urolithiasis. Studying the profile of these patients and the common types of stones will help in offering advice regarding diet and other precautions that can be taken to prevent the occurrence of these renal calculi.

\section{Conflict of Interest: Nil}

Funding: This study was approved by the ICMRSTS programme - reference ID: 2017-00419 and the student received the ICMR grant.

\section{Acknowledgements}

We acknowledge the ICMR for giving students the opportunity to do a research project in their student days. WE are grateful to the Dean and Management of MOSC Medical College for the support and encouragement received to do this study.

\section{References}

1. Romero V, Akpinar H, Assimos DG. Kidney Stones: A Global Picture of Prevalence, Incidence, and Associated Risk Factors. 2010 Reviews in Urology. 12:2/3. e86-96.

2. Sharma SK, Samal IR, Soumya N P. Urinary Stones In Southern India: Biochemical Analysis And Its Clinical Implications. Jan 2015. International Journal of General Medicine and Pharmacy (IJGMP).2015;4:1.

3. Bharathi Pauline Suganthi Vijaya, M. Amirthaveni. A Comparative Study of 24 Hour Urinary Composition between Urinary Stone Formers and Healthy Volunteers. 2007; 44:5:250-260.

4. Gasinska A, Gajewska D. Tea and coffee as the main sources of oxalate in diets of patients with kidney oxalate stones. Roczn, Pzh 2007,58, Nr 1,61-67

5. Pak CY, Sakhee K, Moe O, et al: Biochemical profile of stone forming patients with diabetes mellitus. Urology 61:523-527, 2003
6. Bihl G., Meyers A.: Recurrent renal stones disease-advances in pathogenesis and clinical management. Lancet, 2001, 358, 651-656.

7. Madhvi A, Malhotra SR. Renal Calculi Prevalence, Risk Factors and Dietary Management:AReview.Indian Journal of Nutrition and Dietetics.2016:53:2.228-240.

8. PreziosoD, StrazzulloP,LottiT, Bianchi G, Borghi 1, Caione P et al. Dietary treatment of urinary risk factorsfor renal stone formation. A review of CLU Working Group. ArchivioItaliano di Urologia e Andrologia 2015; 87, 2 105-120.

9. Vasanthamani G, Sushmitha Y 1997. Impact of diet counseling on patients suffering from urinary stones. Indian NutrDietet, 34: 24-28.

10. Carpentier X, Daudon M, Traxer O, Jungers P, Mazouyes A, Matzen G et al. Relationship between the carbonate rate of carbapatite, morphological characteristics of calcium phosphate stones and etiology. 2009. Urology 73:968.

11. Levey AS, Coresh J et al. Clinical Practice Guidelines Clinical Practice Guidelines for Chronic Kidney Disease:Evaluation, Classification and Stratification published by National Kidney Foundation Disease Outcomes Quality Initiative (DOQI). Available at https://www.kidney.org

12. Singh VV, Rai PK. Kidney stone analysis techniques and the role of major and trace elements on their pathogenesis: a review. Biophys Rev (2014) 6:291-310.

13. Taylor EN, Stampfer MJ, Curhan GC. Diabetes mellitus and the risk of nephrolithiasis. Kidney International, 2005, 68:1230-1235.

14. Assimos DG. Diabetes Mellitus and Kidney Stone Formation Rev Urol. 2006;8(1):44.

15. Grases F, Sanchis P, Isern B, Peirello J, CostaBauza A. 2007. Uric acid as inducer of calcium oxalate crystal development. Scandinavian J Urol Nephrol, 41: 26-31.

16. Abate N, Chandalia M, Cabo-Chan Jr. Moe OW, Sakhaee K. The metabolic syndrome and 
uric acid nephrolithiasis: Novel features of renal manifestation of insulin resistance. Kidney International. 2004;65:386-392.

17. Strazzullo P, Barba G, Vuotto P, et al. Past history of nephrolithiasis and incidence of hypertension in men: a reappraisal based on the results of the Olivetti prospective heart study. Nephrol Dial Transplant. 2001;16: 2232-2235 .

18. Boyd C, Wood K, Whitaker D, Assimos DG. The influence of metabolic syndrome and its components on the development of Nephrolithiasis

19. Mehta RL, Kellum JA, Shah SV et al. Acute Kidney Injury Network: report of an initiative to improve outcomes in acute kidney injury. Crit Care 2007; 11: R31

20. Lopes JA, Jorge S. In Depth Review of the RIFLE and AKIN classifications for acute kidney injury: a critical and comprehensive review. Clin Kidney J. 2013; 6: 8-14. 